\title{
Educomunicación y Buenas Prácticas en los nuevos escenarios tecnológicos: Análisis del Caso Gallego (España)
}

\section{Educommunication and Good Practices in the new technological scenarios: Analysis of the Gallego case (Spain)}

\author{
Mari-Carmen Caldeiro-Pedreira ${ }^{1}$ \\ https://orcid.org/0000-0003-0160-3682 \\ Universidad de Santiago de Compostela, España \\ Ignacio Aguaded ${ }^{2}$ \\ https://orcid.org/0000-0002-0229-1118 \\ Amor Pérez-Rodríguez ${ }^{3}$ \\ https://orcid.org/0000-0001-8312-5412 \\ Universidad de Huelva, España
}

Recibido: 06-02-2019

Aceptado: 28-03-2019

\section{Cita Recomendada}

Caldeiro-Pedreira, M.C., Aguaded, I. \& Peréz-Rodríguez, A. (2019) Educomunicación y Buenas Prácticas en los Nuevos Escenarios Tecnológicos: Análisis del Caso Gallego (Espańa) Hamut'ay, 6(1), 96-111.

http://dx.doi.org/10.21503/hamu.v6i1.1577

\section{RESUMEN}

El uso y acceso a las tecnologías de la información y comunicación se ha convertido en una realidad. Actualmente hay casi más teléfonos que personas, además desde edades tempranas se utilizan múltiples pantallas y se accede a Internet. Frente a esta realidad, conviene reflexionar sobre la necesidad de que dos disciplinas de antaño separadas: la educación y la comunicación, aúnen sus esfuerzos para que la totalidad de la ciudadanía esté alfabetizada. En este sentido, resulta fundamental que no solo el profesorado sino también las familias alcancen un mínimo desarrollo de la competencia digital que les permita enseñar a los menores a utilizar adecuadamente los dispositivos digitales. Asimismo, y pese a la inexistencia de políticas educativas claras que amparen la educomunicación como un contenido curricular ni en el contexto español ni en el gallego, se cuenta con iniciativas que responden a los objetivos marcados por el informe DIGCOMP y por el Marco Común de Competencia Digital Docente. Por lo que, en esta investigación con un diseńo de estudio analítico-descriptiva documental, se tuvo como objetivos el caracterizar el término educomunicación en relación con el desarrollo de la competencia digital, así como el analizar la legislación educativa, concretamente del

\footnotetext{
1 Doctora en Comunicación y Educación, docente en la Universidad de Santiago de Compostela (España) Docente en universidades latinoamericanas (Chile y Ecuador). Investigador en el Centro de Estudios de Educación Superior (República Checa).Miembro del consejo asesor y revisor científico de revistas nacionales e internacionales. E-mail: mcarmen.caldeiro@usc.es

2 Catedrático de Educación y Comunicación de la Universidad de Huelva. Es presidente del Grupo Comunicar, un colectivo de alfabetización Mediática de larga trayectoria en España. Jefe del equipo de investigación de Ágora que forma parte del Plan de Investigación de Andalucía (HUM-648), director del Máster Internacional de Comunicación y Educación (UNIA, UHU) y Coordinador del Programa Interuniversitario de Doctorado en Comunicación (UHU, UMA, UCA, UHU).E-mail: aguaded@uhu.es

3 Profesor del Departamento de Filología de la Universidad de Huelva. Miembro del Grupo de Investigación Agora, Grupo Comunicar y Alfamed. Editor asistente de Comunicar Journal y editor de Universitas Journal of Social and Human Sciences (Universidad Politécnica Salesiana, Ecuador). (h-index, 16). Lidera proyectos de investigación nacional e internacional sobre alfabetización mediática y narrativas digitales. E-mail: amor@uhu.es
} 
contexto gallego en España y revisar buenas prácticas existentes para desarrollar la conciencia crítica, autónoma y responsable a edades tempranas.

Una formación que no está regulada como tal ni en el contexto español ni en el gallego. Pese a todo, existen buenas prácticas que, desde el ámbito educativo se están llevando a cabo. Las conclusiones apuestan por una tarea conjunta entre el trabajo de la escuela y la familia que han de favorecer la alfabetización de los menores.

Palabras Clave: Escenarios tecnológicos, educomunicación, educación infantil, buenas prácticas

\section{Abstract}

The use and access to information and communication technologies have become a reality. Currently, there are almost more phones than people. Multiple screens are used and the Internet is accessed from an early age. Faced with this reality, it is convenient to reflect on the need for two formerly separate disciplines: education and communication, to combine their efforts so that the entire citizenship is literate. In this sense, it is essential that not only teachers but also families achieve a minimum development of digital competence that allows them to teach children to properly use digital devices. Likewise, and despite the lack of clear educational policies that assure educommunication as a curricular content neither in the Spanish nor in the Galician context; there are still initiatives that respond to the objectives set by the DIGCOMP report and the Common Digital Competence Framework for Teachers. For this reason, in this research with a documentary descriptive analytical study design, the objectives were to characterize the term educommunication in relation to the development of digital competence, as well as to analyze educational legislation, specifically in the Galician context in Spain and to review existing good practices to develop critical, autonomous and responsible awareness at an early age. An area that is not regulated as such neither in the Spanish nor in the Galician context. In spite of everything, there are good practices that are being executed from the educational field. The conclusions convey a joint task between school and family that benefit the literacy of children.

Keywords: Technological scenarios, educommunication, children education, good practices

\section{INTRODUCCIÓN}

La ciudadanía en general y, de forma concreta los menores utilizan los medios de comunicación para entender el mundo, y buscar modelos con los que se identifiquen (Sahuquillo, 2007; Caridad-Sebastián \& Ayuso-García, 2011). Una realidad que, pese a la existencia de desigualdades a nivel planetario en el acceso a Internet, conforme avanza el tiempo, comienza a disiparse (Pérez-Gutiérrez \& Florido-Bacallao, 2011).
Además de la brecha de acceso, la de uso y la de calidad de uso (Mendoza-Ruano \& Caldera-Serrano, 2014) focalizan la atención dado que no sólo impiden la inclusión digital, sino que favorecen la discriminación socio-cognitiva al tiempo que interfieren en el desarrollo de relaciones tanto horizontales como verticales. Tales desigualdades permiten que se ignoren gran parte de las posibilidades que ofrecen los medios (Carlsson, 2013). Especialmente en el caso de Internet, se obvian potencialidades como son la adquisición y crea- 
ción de conocimiento o la posibilidad de que se comparta con fines no lúdicos ni perniciosos. En este sentido, resulta clave una implicación marcada por parte de la familia y de los educadores, agentes básicos que, de forma marcada a edades tempranas, han de responsabilizarse y promover una educación mediática que permita a los menores a mantener un rol participativo, reflexivo y comprometido ante los medios.

En esta línea, deben primar y fortalecer el desarrollo de la competencia mediática, una tarea que compete no solo a las familias sino además a los docentes; en este sentido, resulta fundamental conocer factores tan relevantes como: la necesidad de poder acceder a los recursos, tecnologías y materiales audiovisuales; el desarrollo profesional de manera continuada que permite abordar los cambios y tener respuestas adecuadas a las nuevas demandas; la colaboración entre familias, estudiantes, docentes, administradores y profesionales de los medios, para compartir conocimientos y experiencias; y por último, la necesidad de investigar sobre alfabetización mediática e informacional. La totalidad de elementos harán posible la continuidad y el avance alrededor de las mejores prácticas de alfabetización, entendida esta última como la disciplina que ofrece conocimientos no solo instrumentales sino también axiológicos y críticos que empoderan al sujeto para actuar de forma responsable en la sociedad digital.

En este contexto, conviene señalar que la presencia de los medios no conlleva la adquisición de la competencia mediática, entendida esta última como la habilidad que contribuye a la alfabetización digital, es decir, la formación necesaria para afrontar los cambios que suponen el uso masivo en las diferentes facetas de la vida de las tecnologías de la información y la comunicación, (Hobbs \& Coiro, 2019). Esta competencia resulta imprescindible en el ecosistema mediático más reciente, y está referida a la habilidad que prepara a la ciudadanía para ser crítica, activa y responsable ante los contenidos audiovisuales que recibe (Aguirre-Sala, 2013; Ballesteros-Velázquez, 2010)

En esta línea, resulta clave apelar a la responsabilidad de la institución educativa y la sociedad tecnológica, una necesidad que se acrecienta cuando las redes y la tecnología están avanzando de forma continua y constante. Este avance lleva intrínseca una complejización de la totalidad de aspectos derivados de la era digital (Ranzólin, 2018) y demanda la conversión del usuario en "prosumidor" de contenidos (Carrero \& Contreras, 2014). En este contexto sociocomunicativo resulta imprescindible el alcance de conductas responsables que se desarrollen con base en las competencias digitales y mediáticas que capacitan al usuario para la adecuada creación de contenidos críticos, responsables y creativos. La tarea de producción constructiva y autónoma inicialmente se consideraba propia de emisores críticos (Aguaded, 2012; Gozálvez \& Contreras, 2014) si bien no es exclusiva de los emisores, sino que corresponde también a los productores, es decir el conjunto de usuarios digitales que deben convertirse en prodiseñadores de contenidos (Hernández-Serrano, Renés-Arellano, Graham \& Greenhill, 2017).

Con el fin de alcanzar este objetivo resulta fundamental la integración de los medios en la enseñanza, una inmersión que ha de producirse desde edades tempranas y que debe realizarse desde una doble óptica: como recurso e instrumento del aprendizaje, ya que se relaciona no solo con el alumnado sino también con su forma de aprender,-semejante a la que se produce fuera del ámbito escolar- y por otra parte, como objetivo mismo del aprendizaje, al ofrecer instrumentos que posibiliten la interpretación y procesamiento de los contenidos que reciben a través de los medios. Con todo ello se auspicia favorecer el desarrollo del pensamiento reflexivo tan necesario en la sociedad de la información y del conocimiento (Ortega-Ruiz, Casas \& Del Rey, 2014). Por todo ello, resulta fundamental considerar nuevos retos culturales, sociales y educativos que permitan la introducción de formas de enseñanza que capaciten al usuario, desde edades tempranas para enfrentarse a la comprensión, creación, gestión y distribución de contenidos, una formación que ha de conducir a la convivencia tanto en el ambiente presencial como en el virtual. (Sánchez-Teruel \& Robles-Bello, 2016).

En este contexto, la educación se enfrenta a nuevos retos adquiriendo un papel clave como mediadora, para ello debe apoyarse en el uso de las nuevas técnicas y estrategias metodológicas, sin 
dejar de lado la adquisición de conocimientos. En definitiva, ha de superarse la mera transmisión vertical central y unidireccional de contenidos; en el contexto audiovisual y digital más reciente, la educación debe cumplir una función comunicativa, horizontal, descentralizada, multidireccional e interactiva. Este cambio define la necesidad de reflexión sobre la transformación de la educación, en general y de forma particular la educación mediática que se propone como resorte para empoderar, no solo al alumnado sino además al profesorado. Asimismo, tal necesidad justifica el hecho de que antes de entrar en la escuela, los menores toman contacto con los medios adquiriendo de este modo, una alfabetización informal básica. Teniendo presente estos hechos, la educación formal no debe alejarse de la realidad y que el profesorado ha de contar con las habilidades necesarias para no incurrir en acrecentar la brecha digital que puede establecerse entre el ambiente escolar y el social de donde proviene el alumnado (Chamorro-Crisaldo, 2018). Para ello, se propone que desde la escuela se apueste por la educación que tiene como centro el aprendizaje significativo que se construye sobre destrezas ya adquiridas y la alfabetización mediática del alumnado que subraya el potencial educativo de las TIC en los contextos intra y extra escolares.

La realidad justifica que la investigación que se presenta se apela al uso de buenas prácticas como forma de desarrollo de la expresión crítica desde edades tempranas. Una expresión que se va cimentando durante toda su vida y que permitirá el alcance de la competencia critica, necesaria en una sociedad hipercomunicada, (García-Avila, 2017; Johnson et al, 2016). Este desarrollo se prevé creciente y continuo a lo largo de la vida de los más pequeños, por ende, se apela al "life long learning» (Longworth, 2005) que permitirá el alcance de la competencia crítica (Caldeiro \& Aguaded, 2015; Tirado \& Ventura, 2009) propia de la ciudadanía que convive en la sociedad hipercomunicada (Regil, 2014) y propia de quienes deben desarrollar nuevas miradas (Cabero \& Marín, 2014).

En la sociedad líquida (Bauman, 2004) el conocimiento se ha vuelto inestable y dinámico (Cabero, 2018) por tanto resulta necesario el desarrollo de nuevas formas de aprendizaje y es imprescindible disminuir la brecha de conocimiento que irremediablemente se establece entre la educación formal que se imparte en las instituciones educativas y la informal a la que, desde edades tempranas estamos expuestos. La realidad justifica el interés por caracterizar el término educomunicación en relación con el desarrollo de la competencia mediática que ha de alcanzarse mediante la adecuada alfabetización digital y cognitiva.

Además, centra el interés el análisis de la legislación educativa, concretamente del contexto gallego en España y revisar buenas prácticas existentes para desarrollar la conciencia crítica, autónoma y responsable a edades tempranas.

\section{Educomunicación y alfabetización en el contex- to digital actual}

Educación y comunicación son dos disciplinas que inicialmente discurrían de manera independiente si bien, conforme se ha ido complejizando el entramado socio-comunicativo, la situación se ha visto modificada de forma determinante. Para (Barbas, 2012; Gregorio-Chaviano, 2018) es un campo interdisciplinar y transdisciplinar que integra dos disciplinas la educación y comunicación. En este sentido, desde finales de la década de los ochenta del pasado siglo la UNESCO ha comenzado a preocuparse por políticas de comunicación en diferentes países. Asimismo, pedagogos como Freinet a comienzos del siglo pasado han apostado por la enseñanza de forma activa. En este sentido, el autor ha sido pionero al sugerir la introducción de la comunicación en el ámbito educativo con el fin de transformar el método memorístico. De esta forma, se ha situado como uno de los precursores a la hora de proponer una nueva forma de enseñar similar a la que hoy en día se demanda. Posteriormente, continuando con esta misma filosofía Freire (1997) se ha referido a la post-alfabetización, un aspecto destacado puesto que, según indica, más allá de la transmisión de conocimientos y la acumulación de los mismos, es necesaria su comprensión.

En este sentido, es posible afirmar de forma rotunda que estos objetivos se adelantaban a aquellos propios de la sociedad digital en el sentido de 
que demandaban un cambio hacia un rol activo del alumnado. Asimismo, apuntaban una necesidad que a lo largo de los años se confirma, el hecho de que el profesorado se convierta en un guía, un mentor que ayuda al discente a construir su propio conocimiento y a crecer en el mismo. Un hito que requiere de nuevas formas de enseñanza, aplicación de estrategias y metodologías que sirvan para poner en marcha buenas prácticas en las cuales la tecnología y el trabajo activo, colaborativo y participativo alcanzan un peso fundamental.

En esta línea, diferentes organizaciones de carácter internacional como es el caso de la UNESCO establecen, según puede leerse en su página web (https://bit.ly/2TKTTzP), la necesidad de empoderar a la ciudadanía a través de diferentes alfabetizaciones que han sido definidas, en diversas ocasiones, como múltiples (Area M. \& Ribeiro P., 2012; Tyner, Gutiérrez \& Torrego, 2015). Se trata de conocimientos que permitan avanzar de lo sólido a lo líquido. Una necesidad de la que debe hacerse eco, de forma indiscutible, la educación, tanto del alumnado como la del propio profesorado (Bergomás, 2019; Fernández, 2015). Sin duda alguna, el profesorado no puede permanecer ajeno a los cambios derivados de la ingente cantidad de tecnología que, de manera exponencial, prolifera en el ecosistema audiovisual más reciente.

Actualmente, la mayor parte de las comunicaciones son mediadas por tecnologías, por ello la educación mediática adquiere un valor clave en el contexto académico y el social en general. Esta necesidad se acentúa en un momento en el cual asistimos al "desarrollo de procesos educativos, mediante los cuales las personas se dotan de herramientas básicas para manejar automáticamente y con consciencia crítica la información y la cultura de la sociedad en la que se encuentran inmersos" (Rodríguez-Fueyo, 2008). En este contexto, resulta necesario focalizar las energías en la formación ciudadana en materia mediática y evitar así, caer en la bruma de la ambigüedad extrema y generalizada Buckingham (2005). Además de ello, "La educación mediática es necesaria para alfabetizarse y dialogar con los medios para que los discentes interaccionen entre sí y también con el profesorado" (Hergueta, 2017, p.21). Esta alfabetización corresponde por partida igual a la edu- cación y la comunicación que existen de forma indisoluble. Ambas disciplinas y sus profesionales han de favorecer y promover el alcance de "visiones más amplias y reflexivas y trazar recorridos complejos es tarea de educadores, con una mirada democrática y un espíritu de aprendizaje y de innovación que nos acerque y no nos aleje" (Quiroz, 2010 , p. 202). En definitiva, la educación mediática y la alfabetización digital deben auspiciar el desarrollo de personalidades autónomas, responsables y críticas. Sujetos capaces de comprender y diseñar contenidos audiovisuales que subrayen el valor de la interrelación social y cívica.

Competencia digital y formación docente para el alcance de "prosumers" críticos

Con el fin de que la ciudadanía en general y los más jóvenes en particular superen la "infoxicación”- sobrecarga informativa que está expuesto el usuario-, (Aguaded, 2014) y, se comuniquen e interrelacionen en el contexto digital surge la necesidad de alcance de la competencia digital. De forma general se entiende esta como una habilidad de carácter teórico-práctico que busca no solo capacitar para localizar información y seleccionarla, sino además para comunicarla de modo adecuado. Esta competencia pretende además que quienes la desarrollen sean capaces de producir contenidos audiovisuales de forma segura y han de saber resolver algún problema con tal difusión y producción. En definitiva, siguiendo el Marco de Competencia Digital Docente (INTEF, 2017), un ciudadano competente digitalmente debe desarrollar los conocimientos que le acreditan para estar al menos en el nivel básico de cada una de las 5 áreas que la componen. Esta acreditación de conocimientos permitirá, no solo a nivel español sino también europeo, contar con las políticas necesarias para, según señala el Informe DigCompedu (2018), "implementar herramientas regionales y nacionales y programas de capacitación» adaptadas a los diferentes niveles de desarrollo».

De este modo, y teniendo en cuenta que los nativos e inmigrantes digitales (Prensky 2001, 2005) deben abandonar el papel de consumidores pasivos y ser capaces de afrontar la recepción de contenidos audiovisuales de forma crítica se propone 
su empoderamiento con el fin de que se conviertan en "prosumidores" activos (Sachez-Carrero \& Contreras, 2012) o más bien en "prodiseñadores de contenido" (Hernández-Serrano, Renés-Arellano, Graham \& Greenhill, 2017).

Para que este objetivo se alcance resulta clave el papel de las familias como agentes formativos fundamentales en la sociedad digital. Desde esta perspectiva, tan importante es la labor de la escuela, como lo es la de las familias, pues en ausencia de pautas educativas, constructivas y orientadoras, surgen situaciones indeseadas que perjudican el bienestar emocional de los más jóvenes y las relaciones con sus iguales. Por tanto, se hace imprescindible alcanzar un equilibrio que además de tratar de evitar los riesgos busque apoyar actuaciones que supongan compartir tiempos y criterios en los ámbitos familiar y escolar "para hacer más potentes las relaciones, más intensos los conocimientos y más adecuadas las comunicaciones, para construir la ciberconvivencia en la red» (Avilés-Martínez \& García-Barreiro, 2018, p.6)

A la luz de los hechos, la legislación educativa contempla la participación familiar como un elemento fundamental en el proceso de aprendizaje. Concretamente en el ámbito gallego, al que nos referimos en esta investigación, en el decreto 330/2009, de 4 de junio, por el que se establece el currículo de la educación infantil en la Comunidad Autónoma de Galicia se hace referencia al proceso educativo de esta etapa y a la participación y colaboración familiar. En este contexto conviene hacer hincapié en uno de los factores más influyentes en las relaciones interfamiliares, la presencia y el uso de los medios de comunicación y las nuevas tecnologías de la información, ya que "la convergencia ha dado pie a un enriquecimiento progresivo en cuanto a la cantidad de dispositivos, mecanismos y formas de expresión que acaban estando a disposición de los ciudadanos y ciudadanas" (Ferrés, 2008, p.66).

La nueva generación de niños y jóvenes, no posee los mismos lenguajes de comunicación que sus progenitores, ya que viven y se comunican chateando, descargando canciones, compartiendo información, navegando por Internet, entre otros, mientras que sus padres/madres aprenden de una manera más paulatina a conocer las nuevas tecnologías y posteriormente a emplearlas. Por cuestiones económicas en gran parte de los casos y de poco conocimiento en otros. De forma general los adultos quedan desvinculados de las apetencias y necesidades de los menores, esto, causa en la educación familiar inquietudes, desequilibrios y problemas, ya que los adultos no conocen las verdaderas necesidades de sus hijos e hijas, y de este modo, es más complicado poder ayudarles o apoyarles en su aprendizaje. En este contexto se justifica la falta de comprensión de los mayores hacia la afición que sus hijos demuestran por determinados modos de estudiar o divertirse.

En esta línea resulta importante destacar que, en la mayor parte de los casos, las personas adultas no están preparadas para leer, entender, comprender, utilizar y cuestionar el lenguaje icónico, lo que dificulta la enseñanza de sus menores a encarar el mundo de las nuevas tecnologías de la educación.

Por todo ello, se infiere básico e imprescindible que los padres y madres tomen conciencia y busquen subsanar sus carencias formativas. Carencias derivadas del hecho de que las familias no se involucran en el acercamiento por parte de sus hijos a las nuevas tecnologías convirtiéndolos de este modo en huérfanos digitales (Junta de Andalucía, 1987, p.3). A la luz de estos hechos resulta preocupante la situación ya que, sin un referente de comportamiento claro, los menores no sabrán enfrentarse a los dilemas que se les planteen. Además de ello, serán más propensos a desarrollar hábitos de convivencia digital poco saludables $y$, estarán más expuestos a potenciales riesgos que no favorecen la e-inclusión.

Con el fin de frenar, en la medida de lo posible, tales riesgos y dificultades se propone la implicación de las familias, ya que, es en este contexto donde el aprender tiene un significado importante y especial que hay que entender muy bien para darse cuenta del valioso papel que desempeña en la vida digital. En este sentido no se puede olvidar que en el contexto social más reciente la mayor parte de los niños y adolescentes, pasan más tiempo con el profesorado y con los medios de comunicación que con su familia, por lo que es importante que el tiempo que estén con la familia, ésta, pueda 
educarles mediáticamente, ya que su responsabilidad es en primer lugar la aceptación de los medios y seguidamente la utilización que se hace en ellos con finalidad de ser un soporte para el cambio social. En cualquier caso, se trata de una tarea múltiple donde la familia educa para la participación, siendo los medios de comunicación y las nuevas tecnologías de la información apoyos clave para alcanzarlo, ya que, "la socialización de los jóvenes por los medios sociales de comunicación es un fenómeno irreversible“ (Frau-Meigs 2011).

Por todo esto, el alcance de niveles elevados de competencia digital se erige como una necesidad clave que deben asumir los diferentes agentes formativos sean o no académicos. Esta realidad certifica el hecho de que "los adultos juegan un papel muy importante en tres aspectos principales: como formadores o responsables directos de su alfabetización digital; como acompañantes o referentes a los que los nińos pueden pedir ayuda; y como ejemplo de modelos de comportamiento» (Vélez, Napal \& Mendioroz, 2018). Por tanto, el desarrollo de la competencia digital por parte de los agentes de referencia contribuirá de forma clave al empoderamiento y alcance de la ciudadanía mediática prosumidora (García-Ruiz, Ramírez \& Rodríguez, 2014). Asimismo, permitirá, aprovechar la tecnología disponible con el fin de abrir la mente y aprender cosas nuevas (especialmente en un momento en el que la red nos acerca a diferentes herramientas y recursos, por ejemplo, hobbies. Por tanto, puede afirmarse que, la totalidad de materiales disponibles han de ser utilizados como herramienta para enseñar a los menores y para ayudarlos a sobrevivir y desarrollarse en la sociedad digital.

Teniendo como referente el título de este epígrafe no se puede dejar de lado el papel del profesorado y la imperante necesidad de que desarrollen niveles óptimos de competencia digital que les permita enfrentarse a los retos de la sociedad digital. En definitiva, la tarea formativa corresponde en primera instancia a las familias seguido de los educadores, es por esto que, "Tanto las familias como los educadores precisan ser competentes mediáticamente para poder ser un modelo personal, profesional y social ante los menores sobre cómo acceder reflexiva y constructivamente a la información y el conocimiento a través de los medios» (González-Fernández, Ramírez-García \& Salcines-Talledo, 2018, p. 301).

De lo normativo a lo práctico: ¿contamos con experiencias reales?

Realizada la justificación y argumentación que certifica la necesidad de intervención educativa y formación integral por parte de la ciudadanía en general conviene revisar aquellas prácticas que, pese a no estar regulado en la legislación educativa de forma explícita, se están desarrollando en pro de la alfabetización mediática de los menores. Un alcance que se realiza teniendo en cuenta las propias necesidades de los menores.

Entre otras experiencias en el contexto español se cuenta con diferentes Blogs y actividades tales que se enmarcan en el contexto socio-digital al que se ha hecho referencia a lo largo del estudio. En este contexto, a la institución educativa le corresponde educar a un alumnado eminentemente digital para formar una ciudadanía prosumidora. Todo ello en un contexto donde adquieren gran importancia las experiencias mediáticas que, a edades tempranas, ya han experimentado previamente dado que, "el aprendizaje se logra de forma tanto explícita como implícita, gracias a un proceso en el que los aprendices son capaces de relacionar conocimientos previos con la experiencia en la resolución de problemas reales» (De la Fuente, 2014, p.454). En este contexto, resulta imprescindible la práctica de la educación mediática en las aulas, estando ésta más próximo al desarrollo de un currículo bimodal (Marqués, 2016).

En este ambiente y pese a las marcadas carencias existen algunas experiencias que pueden verse en la selección de recursos presentes en la Tabla 1 .

Tabla 1

Selección de recursos y actividades

\begin{tabular}{ll}
\hline \multicolumn{1}{c}{ Recursos } & \multicolumn{1}{c}{ Actividades } \\
\hline Web o blog & - EvoluTICvos \\
Proyectos & - El gran libro viajero \\
& - Robot Cubetto \\
Propuesta Metodológica & - Eu cociño, ti cociñas \\
\hline
\end{tabular}

Fuente: elaboración propia 2019 
Para conseguir una formación integral acorde a los intereses y necesidades de los más jóvenes, resulta clave introducir la educación mediática en la etapa de educación Infantil, esta introducción se realiza, como puede apreciarse en la tabla 1 , de diversas formas.

En primer lugar, se pueden diferenciar varios ejemplos de diversos tipos de prácticas educativas para el nivel de Educación Infantil. Las prácticas educativas se entienden como experiencias cuyo nombre surge en el contexto de la política y se vincula al ámbito educativo y en concreto a las prácticas docentes que integran recursos digitales en software libre y redes de comunicación para crear nuevos contenidos y formas de organización escolar. Las buenas prácticas "buscan además promocionar otros tipos de actividades educativas y fomentar estrategias de trabajo colaborativo (De Pablos, \& Jiménez, 2007, p.18), y se entienden como diferentes recursos que no necesariamente tienen que identificarse con actividades a realizar en el aula con los niños y niñas, sino que son proyectos en los que el uso de la tecnología es la materia dominante.

En este sentido la revisión nos muestra diferentes prácticas que se están desarrollando en el contexto espańol y también en el gallego, describiendo a continuación estos recursos.

\section{Blog EvoluTICvos}

Proyecto integral, integrado e integrador, del CEIP Pasico Campillo, en Lorca Murcia. En el que se busca integrar las TIC en el aula de una manera creativa, competencial, funcional y significativa en todas las áreas curriculares. A través de este blog (https://bit.ly/2SIdFet) se muestran las distintas actividades que en esta escuela se realizan con TIC, utilizándolo así, como método de formación para el profesorado.

Esta iniciativa busca sumistrar ayuda tanto a los niños y niñas como a los maestros y maestras, proporcionando así toda la información necesaria para ambos.

Además de los Blog existen a nivel nacional, en el contexto español, diferentes proyectos que persiguen el mismo objetivo, entre otros destacan:

\section{Proyectos}

\section{a. El gran libro viajero}

Iniciativa que surge del blog La Eduteca, un proyecto en el que participan varios colegios de diferentes ciudades de España, recogiendo la riqueza cultural, artística y patrimonial. El principal objetivo de "El Gran Libro Viajero" (https:// bit.ly/2HbtlpA) es potenciar la creatividad y favorecer que el alumnado se familiarice con el conocimiento y la importancia de la cultura o la tradición oral. Se trata de estimular a jóvenes para que participen como gestores de ideas para de este modo, desarrollar un proyecto que pretende, por un lado, enriquecer la cultura y su vocabulario, y por otro, que descubran y conozcan nuevas formas de aprender.

Cada uno de los centros que participa en este proyecto, recibe el libro para trabajar con el alumnado de su clase, a través de la presentación del mismo y seguidamente de la escritura de unas 3 páginas en las que discentes y profesorado tendrán que describir su colegio, su ciudad, o su cultura y que podrán realizarlo de manera libre, es decir, usando pictogramas, dibujos, textos o fotografías.

La estancia del libro en el colegio, junto con las actividades realizadas, es grabada y fotografiada, ya que en el momento en el que el libro se va del colegio, los profesores son entrevistados para conocer la experiencia. El seguimiento y conocimiento de la experiencia de cada uno de los colegios, está en el blog del propio libro, haciendo así que tanto alumnos como profesores sean autores y participantes de un proyecto en el que sin duda la inclusión de las TIC es relevante.

\section{b. Experiencias con el robot Cubetto}

Proyecto que surge de la mano del Colegio Base de San Sebastián de los Reyes (Madrid), ya que en él se trabaja la programación y la robótica dentro de la etapa de educación infantil.

Este proyecto, consiste en la utilización de un pequeño robot de madera que se mueve por medio de unas piezas de plástico de colores en un tablero de control separado.

Con este planteamiento, el alumnado de educa- 
ción infantil aprende a trabajar de forma colaborativa, conoce y desempeña diferentes roles dentro del grupo, aprendiendo así, cuáles son las funciones de un diseñador, un programador y un depurador.

La introducción de la tecnología con esta iniciativa ha generado múltiples y diversas actividades en las que se pueden trabajar diversos temas, favoreciendo así la educación en medios basada en el control únicamente del alumnado.

Además de las prácticas a nivel nacional, en el contexto autonómico, concretamente en la Comunidad de Galicia, se están llevando a cabo iniciativas centradas en el aprendizaje teórico-práctico y su aplicación a la vida diaria.

Propuesta metodológica "Eu cocińo, ti cocińas" alimentación saludable y currículo

Llevada a cabo en un aula del $2^{\circ}$ ciclo de educación infantil del CEIP Sigueiro de Oroso (A Coruña) en la que, no solo el alumnado se dedica a aprender de manera descontextualizada las distintas destrezas y contenidos, si no que a través de los alimentos y la elaboración de recetas se desarrollan los distintos aspectos del Decreto 330/2009, de 4 de junio, por el que se establece el currículo de la educación infantil en la Comunidad Autónoma de Galicia. (https://bit.ly/2kcUVVM).

La propuesta metodológica "Eu cociño, ti cociñas" alimentación saludable y currículo, se describe en la Tabla 2 se centra en varias actividades divididas en bloques:

\section{Tabla 2}

Contenidos y actividades que se llevan a cabo con la práctica "Eu cociño ti cociñas"

\begin{tabular}{ll}
\hline \multicolumn{1}{c}{ Contenidos } & \multicolumn{1}{c}{ Actividades } \\
\hline $\begin{array}{l}\text { Recogida, escucha y/o } \\
\text { transmisión de informa- } \\
\text { ción }\end{array}$ & $\begin{array}{l}\text { Cuentos, adivinanzas, poesías, } \\
\text { imágenes, juegos, videollamadas, } \\
\text { escucha de canciones }\end{array}$ \\
$\begin{array}{l}\text { Análisis e interpretación } \\
\text { de datos }\end{array}$ & $\begin{array}{l}\text { Manipulación, degustación, repre- } \\
\text { sentaciones gráficas, entre otras } \\
\text { actividades. }\end{array}$
\end{tabular}

\begin{tabular}{ll}
\hline \multicolumn{1}{c}{ Contenidos } & \multicolumn{1}{c}{ Actividades } \\
\hline $\begin{array}{l}\text { Memorización y aplica- } \\
\text { ción de contenidos }\end{array}$ & $\begin{array}{l}\text { Recetario, dónde recogen recetas, } \\
\text { las estudian y con las selecciona- } \\
\text { das, después escriben las suyas } \\
\text { propias para escribir su "libro de } \\
\text { recetas" }\end{array}$ \\
Puesta en práctica de lo & $\begin{array}{l}\text { Cocinamos, a través de la elabora- } \\
\text { ción de las recetas seleccionadas } \\
\text { aprendido }\end{array}$ \\
$\begin{array}{l}\text { Explicación y puesta en aula. } \\
\text { común (Programa: Eu }\end{array}$ & $\begin{array}{l}\text { Elaboración de las recetas se gra- } \\
\text { bará en vídeo, elaborarán un pro- } \\
\text { cociño, ¿ti cociñas?,) } \\
\text { grama de cocina en el que explica- } \\
\text { rán el proceso de elaboración }\end{array}$ \\
\hline
\end{tabular}

Jornadas de Puertas Abiertas, en la que realizarán las recetas a invitados especiales y todo el proceso es grabado en vídeo.

Fuente: Elaboración propia partiendo de la Buena Práctica "Eu cociño ti cociñas" (2019)

Este planteamiento sigue la filosofía del aprendizaje por competencias, entendidas como habilidades teórico-prácticas (OCDE, 1997), habilidades cuya base se sitúa sobre la construcción significativa del conocimiento que proporciona al alumnado un nuevo modo de aprender que no se limite a recordar nociones, sino se comprendan y apliquen. Esta aplicación se vincula con todo tipo de experiencias, recursos y herramientas para facilitar la comprensión de los diferentes núcleos temáticos propuestos en el currículo Una necesidad que resulta relevante, de forma especial en la etapa de educación infantil, cuando se sientan las bases de los futuros conocimientos en la edad adulta.

\section{Materiales y Métodos}

\section{Participantes}

La población de estudio es el análisis documental basada en una revisión bibliográfica a través de bases de datos tales como: Dialnet, Scielo, WOS, SCOPUS, haciendo un total de artículos 95 encontrados que formaron parte de la población. Los artículos seleccionados han sido mayoritariamente en español si bien se han consultado algunos en lengua inglesa. La selección de la muestra se realizó considerando los siguientes criterios:

1. Aplicación de los descriptores: sociedad digital, educación, educación infantil, legislación, 
buenas prácticas, como criterio de inclusión.

2. Criterio de exclusión de los años 2013-2018, es decir, desde la implantación en España de la LOMCE (Ley Orgánica de Mejora de la Calidad Educativa) hasta la actualidad. Pese a todo, en las referencias figuran materiales anteriores dada la necesidad de justificar la continuidad y origen de los modelos pedagógicos que demanda la sociedad digital.

Una vez realizado el análisis de la población $(\mathrm{N}=95)$ se obtuvo un muestreo no probabilístico, por conveniencia, teniendo en consideración los criterios de inclusión y exclusión, obteniéndose como muestra 54 artículos.

\section{Instrumento}

La recolección de datos se hizo con una rejilla de indicadores, (López, 2016) en el que se seleccionaron tres criterios:

1. Indicadores bibliométricos: publicación por año, base de datos, país de estudio.

2. Términos de búsqueda: Descriptores de la temática a analizar

3. Selección de los documentos: Criterios inclusión y exclusión

Tipo y diseño

La investigación es de tipo descriptiva, ya que se busca caracterizar el término educomunicación en relación con el desarrollo de la competencia mediática que ha de alcanzarse mediante la adecuada alfabetización digital y cognitiva. (Hernández, Fernández \& Baptista, 2014)

Además, centra el interés en analizar la legislación educativa, concretamente del contexto gallego en España y revisar buenas prácticas existentes para desarrollar la conciencia crítica, autónoma y responsable a edades tempranas.

La metodología hace referencia a aspectos de diferente nivel de concreción dentro del proceso de investigación (Montero \& León, 2005), en este estudio se realiza una revisión documental y posterior reflexión sobre los aspectos clave mencionados.

\section{Procedimiento}

El estudio se realizó de una manera secuencial y organizada por etapas. Como se puede observar en la Figura 1.

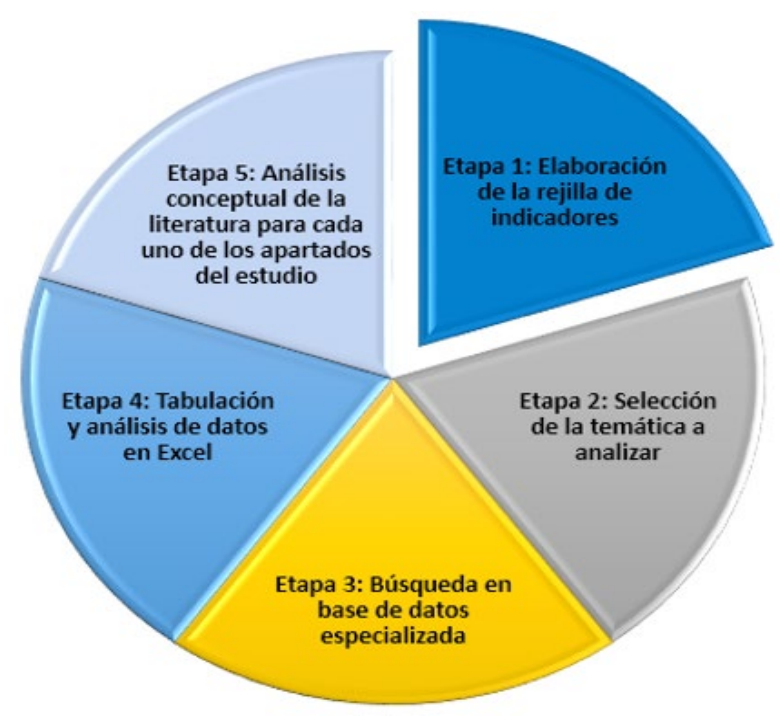

Figura 1

Procedimiento de analisis de la revisión del estudio.

Fuente: Elaboración propia 2019

\section{Resultados}

El análisis de resultados se hizo desde dos vertientes: a) Desde un carácter descriptivo bibliométrico y b) Desde el aspecto conceptual y teórico, como se muestra en la Figura 2.

Las experiencias descritas en los apartados anteriores se realizan en un marco normativo donde la educación mediática queda relegada a la transversalidad y la interdisciplinariedad, especialmente en la legislación educativa de la etapa de Infantil. A Grosso modo puede afirmarse que esta situación se repite tanto en el contexto español, con la entrada en vigor de la LOMCE, como en el gallego puesto que la legislación de la etapa de educación infantil no recoge el término "educomunicación" en ningún caso. En este sentido, conviene señalar que La ley Educativa vigente hasta el año 2013, Ley Orgánica 2/2006 de 3 de mayo de Educación, define en su artículo 6, el currículo entendido como el conjunto de competencias básicas, contenidos, métodos pedagógicos 

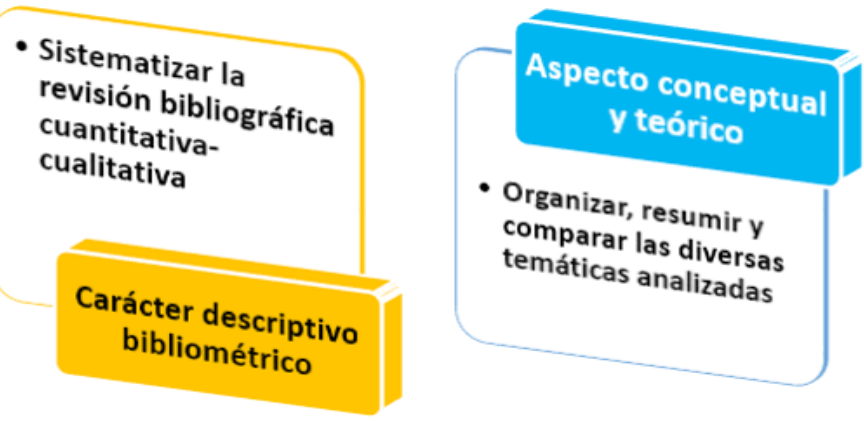

\section{Figura 2}

Análisis de los resultados.

Fuente: Elaboración propia 2019

y criterios de evaluación. Sin embargo, no hace mención a la educación mediática ni en medios ni a la alfabetización tecnológica. Pese a las diferentes modificaciones no se aprecian, en esta línea, cambios notables. La modificación de 2013 afecta únicamente a un artículo que cambia varios artículos de la antigua LOE. Una situación que conduce al desarrollo de la Ley Orgánica 8/2013, 9 de diciembre, para la Mejora de la Calidad Educativa, es decir, la LOMCE. En ésta el currículo de Educación Infantil, está comprendido concretamente por tres áreas de conocimiento: conocimiento de sí mismo y autonomía personal; conocimiento del entorno y el área de lenguajes: comunicación y representación. Se trata de áreas en las que se subraya la necesidad de que, desde el primer ciclo, se haga hincapié en el desarrollo de la autonomía de los niños y niñas, y la creación de los primeros vínculos sociales junto con la conquista del lenguaje, aspectos que adquieren un valor relevante en la sociedad digital donde es necesario desarrollar las competencias necesarias para comprender los contenidos y producirlos de forma responsable y autónoma. En este sentido, además de fomentar una primera toma de contacto con la lectura, la escritura y con experiencias de iniciación temprana en habilidades numéricas básicas, se requiere de un contacto marcado con las tecnologías de la información y la comunicación y la expresión visual y musical. Objetivos que pueden alcanzarse a través del desarrollo de las estrategias y metodologías que recoge la (Tabla 1). Actividades a través de las cuales es posible introducir la alfabetización mediática en la primera infancia de forma divertida a la par que efectiva.
En definitiva, puede afirmarse que, las buenas prácticas educativas, lo son si forman parte de ellas alumnos, profesores y familias, unos y otros han de conseguir desarrollar un nivel adecuado de competencia mediática. Una tarea que, dado su carácter transversal debe desarrollarse entre los ámbitos familiar y escolar puesto que, alfabetizar corresponde en primer lugar a la familia y la tarea debe continuarse en la escuela.

Esa situación no es exclusiva de España, según nuestros datos en Latinoamérica también es preocupación de los investigadores la relación efectiva de la tecnología y la familia en relación con la educación, es así como mostramos en la Tabla 3 las investigaciones orientadas en el tema, cosa que nos invita a seguir impulsando este tipo de iniciativas. Se puede notar en España se realizan aproximadamente $62 \%$ de trabajos de investigación en el tema en estudio en comparación con Latinoamérica en ese año.

Tabla 3

Distribución de publicaciones sobre el tema según la región entre 2015

\begin{tabular}{lcc}
\hline \multicolumn{1}{c}{ Region } & Cantidad de investigaciones 2015 & $\%$ \\
\hline España & 10 & 62 \\
America Latina & 6 & 38 \\
\hline
\end{tabular}

Fuente: Elaboración propia, 2019

En la Tabla 4 se constatan los tipos de trabajos y la cantidad de ellos que realizan en Espańa y latinoamericano. Se aprecia la diferencia en los tipos de trabajos que se desarrollan en cada uno y en cual base de datos fue publicada, tal es el caso de España donde podemos encontrar artículos Latindex, capítulos de libro, acta de congreso y tesis doctoral. A diferencia de los artículos publicados en las bases de datos Scielo y Scopus fueron desarrollados con más éxito en Latinoamérica que España.

La Tabla 4 refleja los tipos de trabajos y la cantidad de ellos que realizan en Espańa y latinoamericano. Se puede notar la diferencia en los tipos de trabajos que se desarrollan en cada uno y en cual base de datos fue publicada, tal es el caso de España donde podemos encontrar artículos Latindex, capítulos de libro, acta de congreso y tesis docto- 
ral. A diferencia de los artículos publicados en las bases de datos Scielo y Scopus fueron desarrollados con más éxito en Latinoamérica que España.

\section{Tabla 4}

Porcentajes del número de publicación según país y tipo de trabajo de las variables de estudio 2017

\begin{tabular}{lclr}
\hline $\begin{array}{c}\text { País de la } \\
\text { publicación }\end{array}$ & $\begin{array}{c}\text { Cantidad } \\
\text { de publica- } \\
\text { ciones }\end{array}$ & $\begin{array}{l}\text { Tipo de trabajo y/o base } \\
\text { de datos }\end{array}$ & $\%$ \\
\hline México & 3 & $\begin{array}{l}\text { Artículo WOS+Scie- } \\
\text { lo+ESCl }\end{array}$ & 18.75 \\
Argentina & 1 & $\begin{array}{l}\text { Artículo WOS+Scie- } \\
\text { lo+ESCl }\end{array}$ & 6.25 \\
Colombia & 1 & Articulo Scopus & 6.25 \\
España & 1 & Artículo WOS & 6.25 \\
España & 3 & Artículo ESCl & 18.75 \\
España & 2 & Artículo Latindex & 12.5 \\
España & 1 & Capítulo de Libro & 6.25 \\
España & 1 & Actas congreso & 6.25 \\
España & 2 & Tesis doctoral & 12.5 \\
Ecuador & 1 & Artículo ESCl & 6.25 \\
\hline
\end{tabular}

Fuente: Elaboración propia, 2019

En la Figura 3 se observa cantidad de los proyectos de investigación relacionado educomunicación y buenas prácticas, los temas de revisión y los reportes de caso que se han desarrollado entre los años 2010 y 2015. Es notable como en el año 2013 los proyectos originales de investigación tuvieron una caída de 11 proyectos en el año 2012 a 8 proyectos en el ańo 2013. En los siguientes dos años hubo en aumento considerable hasta llegar en el 2015 a 14 proyectos. Los temas de revisión en los ańos 2010 y 2011 fueron 3 proyectos por año, un aumento significativo en el año 2012 y 2013 donde duplicaron su cantidad desarrollados y finalizando en los años 2014 y 2015 con 4 proyectos. Los reportes de caso como se puede notar no son muy comunes, en el periodo de 6 años se realizaron solo 4 proyectos.

Se aprecia en la tabla 5 un incremento de aproximadamente un $28 \%$ en cantidad de proyectos que se realizaron desde el año 2010 al año 2015 sin tomar en cuenta el tipo de proyecto. Empezando en el año 2010 con 13 proyectos realizados, al paso de 5 años en el año 2015 se llegaron a desarrollar 18 proyectos.
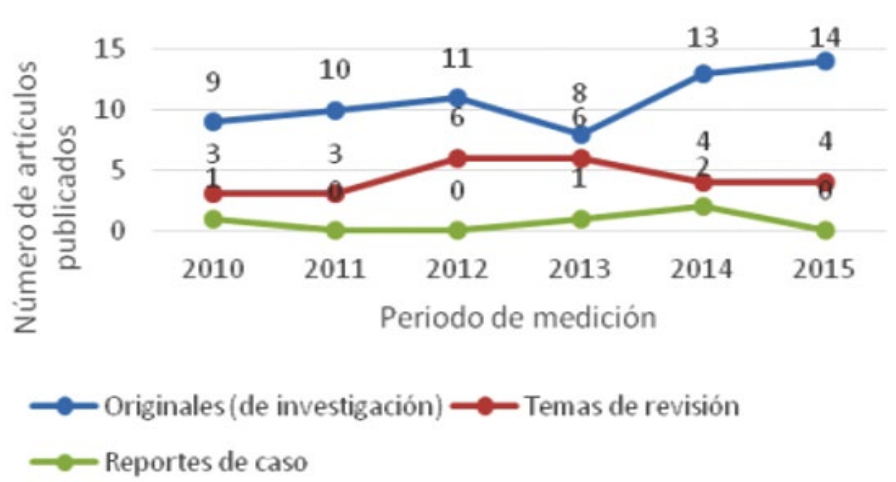

Figura 3

Número de articulos publicados 2010-2015

Fuente: Elaboración propia 2019

Tabla 5

Cantidad de publicaciones por año 2010-2015

\begin{tabular}{cc}
\hline Año & Investigaciones \\
\hline 2010 & 13 \\
2011 & 13 \\
2012 & 17 \\
2013 & 15 \\
2014 & 19 \\
2015 & 18 \\
\hline
\end{tabular}

Fuente: Base de datos Scielo, Scopus, Latindex, WOS.

\section{Discusión y Conclusiones}

Es de resaltar, de acuerdo a lo analizado en la revisión bibliográfica, que la educomunicación se erige como una nueva vertiente en la educación de los niños y jóvenes al ser esta integradora de diversas competencias. Habilidades que los jóvenes han tenido que desarrollar de forma inexcusable. Esta realidad implica el fomento de nuevas políticas públicas acordes con los múltiples cambios generados en este siglo. Un hecho que constata Aparici (2010) al mencionar que la educomunicación contempla múltiples lenguajes y medios, al permitir realizar una comunicación personal, grupal y social que integra una formación con sentido crítico e inteligente frente a los procesos comunicativos y sus mensajes.

En otro orden de hechos y, según se desprende de la legislación educativa vigente en España y en Galicia, hoy en día no existen directrices curriculares concretas ni políticas educativas claras que 
amparen la educación mediática como una disciplina con entidad propia. Tanto en el contexto nacional como en el autonómico, como ha podido apreciarse anteriormente, se están llevando a cabo experiencias que sirven de modelo y que ejemplifican el interés y necesidad de alfabetización por parte de los menores al tiempo que certifican la necesaria formación de los docentes, especialmente los de edades tempranas.

Por otra parte, la escasa cantidad de prácticas educativas que se desarrollan no solo en estos dos ámbitos sino también en los contextos europeo e incluso en el latinoamericano, se requiere de un cambio pedagógico por parte del profesorado que, además de adquirir niveles óptimos de competencia digital ha de enseñar según el modelo de competencias que proponía la OCDE (1997), en este sentido el profesorado debería utilizar, por ejemplo, la gamificación de los contenidos para, por ejemplo, favorecer "el desarrollo de la lectura dentro del entorno mediático y digital» (Torres, Romero, Pérez \& Björk, 2016, p. 37) y para alfabetizar a los discentes según sus necesidades (Peñalva \& De-Casas, 2018). En este contexto el docente ha de modificar su rol de transmisor de contenidos para trabajar con nuevas metodologías, estrategias que contribuyen a reducir la posible brecha digital y por otro lado preparan al alumnado para que pueda enfrentarse a la sociedad donde tendrá que desarrollarse, donde tendrá que ser capaz de encarar situaciones derivadas de un irregular uso de la tecnología tales como diferentes tipos de acoso; así como los diversos tipos de lenguajes y formas de comunicación y aprendizaje que se están dando con el advenimiento de las tecnologías (Fernández, 2015)

Para finalizar, la realización de esta investigación permite señalar, de forma general, las características de las prácticas educativas que están vinculadas con el fin o propósito, "en la acción intencional", el "saber qué hacer". Un hecho que debe tener en cuenta que se convierte en "saber cómo" de forma más directa, es decir, el tener en mente lo que se quiere conseguir estará íntimamente unido al cómo lograr lo que se desea obtener" (Ospina \& Montoya, 2017). Asimismo, se constata la importancia de llevar a cabo prácticas educativas en las que los niños y niñas, no sólo las ejecuten, sino que han de interiorizarlas de modo que la formación en medios no solo sea completa sino también eficaz.

Para ello resulta clave el trabajo conjunto de la escuela y la familia para favorecer una adecuada alfabetización y desarrollo de la competencia digital que capacita, en este caso, al menor para su adecuado desarrollo.

Con el fin de alcanzar este objetivo sería deseable el trabajo conjunto entre ambos agentes educativos y se considera deseable que, por ejemplo, la escuela pudiese proporcionarles a las familias, además de materiales didácticos, un seguimiento detallado de sus hijos acorde a su formación y al alcance y desarrollo de sus conocimientos. Con esta iniciativa se contribuiría a favorecer la conexión y fluidez entre ambas partes y contribuiría además al mejor aprendizaje de los menores. Es también sensible la necesidad de incrementar las iniciativas de investigación en esta área para incrementar la cobertura de esta información y así reconocer y aplicar métodos y herramientas en lengua española, favoreciendo así a España y Latinoamérica. El crecimiento sostenido desde 2015 al 2017 debería continuar influenciado con la masificación de herramientas y equipos portátiles como teléfonos inteligentes y tablets que facilitar el desarrollo de proyectos de investigación orientados a develar el importante número de interrogantes presentes en este nuevo escenario educativo.

En síntesis y como última reflexión, se considera imprescindible un cambio en la metodología, que consiga que la inclusión de las TIC sea completa y absoluta.

\section{Agradecimientos}

Este trabajo se ha elaborado en el marco de Alfamed (Red Interuniversitaria Euroamericana de Investigación en Competencias Mediáticas para la Ciudadanía), con el apoyo del Proyecto $\mathrm{I}+\mathrm{D}+\mathrm{I}$ Coordinado "Competencias mediáticas de la ciudadanía en medios digitales emergentes (smartphones y tablets): practicas innovadoras y estrategias educomunicativas en contextos múltiples"(EDU2015-64015-C3-1-R) (MINECO/ FEDER), la "Red de Educación Mediática" del 
Programa Estatal de Investigación Científica-Técnica de Excelencia, Subprograma Estatal de Generación de Conocimiento (EDU2016-81772REDT), financiados por el Fondo Europeo de Desarrollo Regional (FEDER) y Ministerio de Economía y Competitividad de España. Y en el Marco del I+D de la Universidad de Cantabria "Competencia mediática en la sociedad digital para la participación crítica de la ciudadanía (UC2016-GRE-01)".

\section{REFERENCIAS BIBLIOGRÁFICAS}

Aguaded, I. (2014). From Infoxication to the Right to Communicate. Comunicar, 42, 07-08. https://doi.org/10.3916/ C42-2014-a1

Aguirre-Sala, J. (2013). Nuevos alcances de la participación ciudadana a través de las redes sociales. Culturales, 1(2), 119-150. Recuperado de https://bit.ly/2IOeHG9

Apararici, R. (2010) Educomunicación: Más allá del 2.0., Barcelona, Gedisa.

Area, M. \& Ribeiro, M.T. (2012). From Solid to Liquid: New Literacies to the Cultural Changes of Web 2.0. Comunicar, 38, 13-20. https://doi.org/10.3916/C38-2012-02-01

Avilés-Martínez, J. \& García-Barreiro, J. (2018). Ciberconvivencia y ciudadanía digital. Revista digital de la Asociación CONVIVES. 22.

Ballesteros Velázquez, B. (2010): “Educación y medios”. In Aguado, T. (Coord.): Diversidad e igualdad en educación, pp. 277-316, UNED, Madrid

Barbas, A. (2012) Educomunicación: desarrollo, enfoques y desafíos en un mundo internconectado. Foro de Educación, 10 (14), 157-175.

Bauman, Z. (2004). Modernidad líquida. FCE: Argentina. Recuperado de https://goo.gl/zQL632

Bergomás, G. (2019). Las alfabetizaciones múltiples como eje de la formación docente. Razón y Palabra, 63. Recuperado de https://bit.ly/2tCqwoz

Buckingham, D. (2005). Educación en medios: alfabetización, aprendizaje y cultura contemporánea. Barcelona: Paidós.

Cabero, J. (2018). Tic y educación en la sociedad del conocimiento. In Ortiz-Colón, A. \& Ortega-Tudela, J.M.(cords) (2018). Tecnologías en entornos educativos. Madrid: Paraninfo. PMCid:PMC5920066

Cabero-Almenara, J. \& Marín-Díaz V. (2014). Miradas sobre la formación del profesorado en TIC, Enl@ce. Revista Venezolana de Información, Tecnología y Conocimiento, 11(2). 11-24. Recuperado de https://bit.ly/1G09Ber

Caldeiro, MC. \& Aguaded-Gómez, J. (2015). «Estoy aprendiendo, no me molestes» la competencia mediática como forma de expresión crítica de nativos e inmigrantes digitales. Redes.com, 12. Recuperado de http://goo.gl/2ORkf1

Caridad-Sebastián, M. \& Ayuso-García, M. (2011). Situación de la brecha digital de género y medidas de inclusión en Espa-a. Investigación bibliotecológica, 25(55), 227-252. https://doi.org/10.22201/iibi.0187358xp.2011.55.32973

Carlsson, U. (2013). Los jóvenes en la cultura digital y mediática: perspectivas globales y escandinavas. Analisí Monogràfic 2013. 77-94. Recuperado de https://goo.gl/xnKjoH

CEIP Pasico Campillo, en Lorca Murcia. https://bit. ly/2Opj2Pm

CEIP Sigueiro de Oroso (A Coruña). Recuperado de https://bit.ly/2Tstbvk

Chamorro-Cristaldo, M. (2018). Digital divide, factors affecting your appearance: internet access in Paraguay. Población y Desarrollo, 24(47), 58-67. https://doi.org/10.18004/ pdfce/2076-054x/2018.024(47)058-067

Colegio Base de San Sebastián de los Reyes (Madrid). Recuperado de https://bit.ly/2VCaAyy

Commision European (2018). DigCompEdu. Recuperado de https://bit.ly/2BRoeGD-De la Fuente, J. (2014). Alfabetización mediática: del prosumidor al profesional. Historia y Comunicación Social, 19. pp. 451-464. https://doi. org/10.5209/rev_HICS.2014.v19.45041

De Pablos-Pons, J. \& Jiménez-Cortés, R. (2007). Buenas prácticas con TIC apoyadas en las Políticas Educativas: claves conceptuales y derivaciones para la formación en competencias ECTS, Revista Latinoamericana de Tecnología Educativa, 6 (2), 15-28. Recuperado de https://bit.ly/2w9hkZD

Díaz-Lazo, J. Pérez-Gutiérrez, A. \& Florido-Bacallao, R. (2011). Impacto de las tecnologías de la información y las comunicaciones (Tic) para disminuir la brecha digital en la sociedad actual. Cultivos Tropicales, 32(1), 81-90. Recuperado de https://bit.ly/2tQ3iet

DOG 121. DECRETO 330/2009, de 4 de junio, por el que se establece el currículo de la educación infantil en la Comunidad Autónoma de Galicia. Recuperado de https:// bit.ly/2kcUVVM

Fernández, E. (2015) Educomunicación: Las tecnologías de la información y la comunicación, en la educación. (Tesis de grado), Facultad de Publicidad y RR.PP. Universidad de Valladolid, España.

Ferrés, J. (2008). La educación como industria del deseo. Un nuevo deseo comunicativo. Gedisa: Barcelona. PMCid:PMC2648280

Frau-Meigs, D. (2011). Socialisation des jeunes et éducation aux médias. Toulouse, France: Eres. https://doi. org/10.3917/eres.fraum.2011.01

Freire, P. (1973) ¿Extensión o comunicación? la concientización en el medio rural. México. Siglo veintiuno editores.

García-Ávila, S. (2017). Alfabetización Digital. Razón y Palabra, 21 (98), 66-81. Recuperado de https://bit.ly/2MbFNbj 
García-Ruiz, R., Ramírez, A. \& Rodríguez, M. (2014). Media Literacy Education for a New Prosumer Citizenship. Comunicar, 43, 15-23. https://doi.org/10.3916/C43-201

González-Fernández, N., Ramírez-García, A. \& Salcines-Talledo, I. (2018). Competencia mediática y necesidades de alfabetización audiovisual de docentes y familias españolas. Educación XX1, 21(2), 301-321, https://doi.org/10.5944/ educxx1.16384

Gozálvez, V. \& Contreras, P. (2014): Empoderar a la ciudadanía mediática desde la educomunicación. Comunicar, 42, 129-136. https://doi.org/10.3916/C42-2014-12

Gregorio-Chaviano, O. (2018) Análisis de la educomunicación como campo de investigación en desarrollo: una aproximación bibliométrica. Paper, III Congreso Internacional, Competencias Mediáticas, Medellín, Colombia.

Hergueta Covacho, E. (2017) Educación mediática. Propuesta metodológica para el desarrollo de la competencia mediática en la práctica educativa. Programa de doctorado en comunicación y educación en entornos digitales.

Hernández, R., Fernández, C. \& Baptista, P. (2014). Metodología de la Investigación. México: McGraw-Hill.

Hernández-Serrano, M., Renés-Arellano, P., Graham, G. \& Greenhill, A. (2017). From Prosumer to Prodesigner: Participatory News Consumption. Comunicar, 50, 77-88. https://doi.org/10.3916/C50-2017-07

Hobbs, R. \& Coiro, J. (2019). Design Features of a Professional Development Program in Digital Literacy. Journal 1 of Adolescent \& Adult Literacy, 62 (4). 401-409. https://doi. org/10.1002/jaal.907

INTEF (2017). Marco Común de Competencia Digital Docente. Recuperado de https://bit.ly/2EwAu0O

Johnson, L., Adams Becker, S., Cummins, M., Estrada, V., Freeman, A. \& Hall, C. (2016). NMC Informe Horizon 2016 Edición Superior de Educación. Austin, Texas: The New Media Consortium.Recuperado de https://bit.ly/2H$\mathrm{dbWcW}$

Junta de Andalucía (1978). Educar para proteger. Guía de formación TIC para padres y madres de menores de 3 a 11 ańos. Recuperado de https://bit.ly/2TkzFQQ

Ley Orgánica 2/2006, de 3 de mayo, de Educación. Recuperado de https://bit.ly/2tE8hPC

Ley Orgánica 8/2013, 9 de diciembre, para la Mejora de la Calidad Educativa, LOMCE. Recuperado de https://bit. ly/2hnUxTt

Longworth, N. (2005). El aprendizaje a lo largo de la vida en la práctica. Barcelona: Paidós.

López, A. (2016) Tesis de la actividad científica de la técnica psicológica de rejilla a través de indicadores bibliométricos. (Tesis doctoral). Universidad Nacional de Educación a Distancia, España. Recuperado de https://bit.ly/2WITuW3

Marqués, P. (2016). Síntesis de las investigaciones realizadas entre 2011 y 2016: ¿por qué conviene aplicar el CURRÍCULUM BIMODAL? Recuperado de https://bit.ly/2TfeSye
Mendoza-Ruano, J., \& Caldera-Serrano, J. (2014). Umbrales para la determinación de la brecha digital: comparativa entre regiones desarrolladas. Transinformação, 26(2), 125 132. https://doi.org/10.1590/0103-37862014000200002

Montero, I., \& León, O. (2005). Sistema de clasificación del método en los informes de investigación en Psicología. International Journal of Clinical and Health Psychology, 5 (1), 115-127.

OCDE. (1997). La definición y selección de competencias clave. Recuperado de https://bit.ly/2eILmLD

Ortega-Ruiz, R., Casas, J.A. \& Del Rey, R. (2014). Hacia el constructo de ciberconvivencia. Infancia y Aprendizaje, 37 (3), 602-628. https://doi.org/10.1080/02103702.2014. 957537

Ospina Botero, M. \& Montoya Pavas, E. (2017). Las prácticas educativas con familia desde la escuela. Revista del Instituto de Estudios en Educación y del Instituto de Idiomas Universidad del norte. pp. 31-43

Peñalva, S. \& De Casas, P. (2018). El uso de la gamificación como metodología educomunicativa en el contexto universitario. In Torres, A. \& Romero, LM. (ed.) (2018). Gamificación en Iberoamérica. Experiencias desde la comunicación y la educación. Recuperado de https://bit.ly/2HecqBE-Pe-alva-Vélez.

Peñalva, A., Napal, M. \& Medioroz, A.M. (2018). Competencia digital y alfabetización digital de los adultos (profesorado y familias). International Journal of New Education, 1, 1-13. https://doi.org/10.24310/IJNE1.1.2018.4892

Prensky, M. (2001). Digital Natives, Digital Immigrants. New York: MCB University Press. PMid:11282041

Prensky, M. (2005). Teaching digital natives: partnering for real learning. Recuperado de https://goo.gl/opELD9

Quiroz Velasco, T. (2010). Educar en otros tiempos. El valor de la comunicación. En R. Aparici (Ed.), Educomunicación: más allá del 2.0 (pp.187-203)

Ranzólin, A. (2018). Educomunicación y ciudadanía: Reflexiones sobre responsabilidades compartidas en un mundo digital. Aularia 7 (1), pp. 11-16. Recuperado de https://bit. ly/2UlHhQi

Regil, L. (2014). Cultura digital universitaria. Tesis doctoral. Recuperado de https://bit.ly/2Ulamvc

Rodríguez- Hoyos, C. \& Fueyo, A. (2011). La alfabetización audiovisual crítica en la sociedad de la información. Una experiencia de formación continua. Pixel Bit. Revista de Medios y educación, 39. Pp. 95-107. Recuperado de https://bit.ly/2EapUv7

Sahuquillo, P. (2009). Algunas aportaciones teóricas a la influencia de la televisión en el proceso de socialización de la infancia. Teoría De La Educación. Revista Interuniversitaria, 19(1). Recuperado de https://goo.gl/HDRSuW

Sánchez-Carrero, J. \& Contreras, P. (2012). De cara al prosumidor: producción y consumo empoderando a la ciudadanía 3.0. 14, 10 (3); 62-84. https://doi.org/10.7195/ri14. v10i3.210 
Sánchez-Teruel, D. \& Robles-Bello, M. A. (2016). Riesgos y potencialidades de la era digital para la infancia y la adolescencia. Revista Educación y Humanismo, 18(31), 186-204. http://dx.doi.org/10.17081/eduhum.18.31.1374

Tirado, M.A. \& Ventura, C. (2009) Proposals for the development of critical competence in Physical Education, Cultura y Educación, 21:1, 55-66. https://doi. org/10.1174/113564009787531181

Torres-Toukoumidis, A., Romero-Rodríguez, L., Pérez-Rodríguez, M. A., \& Björk, S. (2016). Development of reading skills through video games: state of the art. Ocnos, 15 (2), 37-49. https://doi.org/10.18239/ocnos_2016.15.2.1124

Tyner, K., Gutiérrez, A. \& Torrego, A. (2015). "Multialfabetización" Sin muros en la era de la convergencia. La competencia digital y "la cultura del hacer" como revulsivos para una educación continua. Revista de currículum y formación del profesorado, 19(2), 41-56. https://bit.ly/2CyLQzS 